\title{
INFLUENCE OF PHYSICO-CHEMICAL PARAMETERS ON SECONDARY METABOLITE PRODUCTION BY MARINE FUNGI
}

\author{
ANUHYA G., JYOSTNA V., ASWANI KUMAR YVV, BODAIAH B., SUDHAKAR P.* \\ Department of Biotechnology, Acharya Nagarjuna University, Guntur, Andhra Pradesh, India 522510 \\ Email: sudhakarpodha@gmail.com
}

Received: 24 May 2017, Revised and Accepted: 22 Jul 2017

\begin{abstract}
Objective: Our study aimed to characterize and optimize the physico-chemical properties which render the high yield of bioactive secondary metabolites from marine fungi and its antibacterial activity against clinical pathogens.

Methods: Bioactive secondary metabolites extracted from marine fungi of Nijampatnam mangroves, Guntur district Andhra Pradesh, India. By using different solvent systems, secondary metabolite was screened for antimicrobial activity against clinical pathogen, Optimization of cultural conditions for maximizing the yield of biomass and compared the yield in different broth media and minimum inhibitory concentration of the optimised compound done for both compounds.

Results: fungal extracts GAPS- 1 and GAPS-2 was affectively extracted in ethyl acetate. Isolated compound showed antibacterial activity highest zone of inhibition against E. coli and least to Pseudomonas. Maximum yield of the compound was achieved in modified potato dextrose broth (PDB) medium containing $20 \% \mathrm{w} / \mathrm{v}$ Potato $2 \% \mathrm{w} / \mathrm{v}$ Dextrose broth containing $1 \% \mathrm{w} / \mathrm{v}$ glucose and sucrose, $1 \% \mathrm{w} / \mathrm{v}$ beef extract and ammonium chloride and $0.01 \%$ mineral salts $\left(\mathrm{MGSO}_{4}\right.$ and $\left.\mathrm{MNSO}_{4}\right)$ at $25^{\circ} \mathrm{C}$ and $30{ }^{\circ} \mathrm{C}$ and $\mathrm{pH} 7.0$ and 8.0 with $144 \mathrm{~h}$ of incubation period. The yield was observed maximum in potato dextrose broth compared to czapek dox broth, sabaraud's broth and nutrient broth and minimum inhibitory concentration of the optimized compound ranges from $250 \mu \mathrm{g}$ for gaps-1 and $300 \mu \mathrm{g}$ for gaps- 2 .
\end{abstract}

Conclusion: Secondary metabolite yield maximum in potato dextrose broth and its potential anti-bacterial activity needs further investigation for pharmaceutical applications.

Keywords: Secondary metabolite, Anti bacterial activity, Optimization

(c) 2017 The Authors. Published by Innovare Academic Sciences Pvt Ltd. This is an open access article under the CC BY license (http://creativecommons.org/licenses/by/4.0/) DOI: http://dx.doi.org/10.22159/ijcpr.2017v9i5.22152

\section{INTRODUCTION}

Natural environment is still the most important contributor of novel drugs in the face of development of combinatorial chemistry, which quickly generated thousands of new chemicals [1]. Microbial sources from marine environments are prolific producers of natural antibiotics, with massive prevalence of drug resistant towards pathogenic microorganisms. It is crucial to search for novel antibiotics with wide applications in chemotherapy, plant pathology, food preservation, veterinary medicine, biotechnology and molecular biology. The applicable antibiotics may affect the screening of bioactive compounds, with selective and sensitive bioactivity detection methods of their importance [2]. The biosynthesis of antibiotics like other microbial metabolites is determined by a number of regulatory factors [3]. Including growth conditions, carbon, nitrogen, mineral salt levels and physical parameters like temperature, $\mathrm{pH}$ and agitation during production. These conditions may vary from species to species from many of the microbes that live in extreme environments like high temperatures, high salt concentrations, low $\mathrm{pH}$, and high radiation etc, influence the specifically fungal growth and metabolite productions. Usually the bioactive metabolite production from microorganisms depends on their special adaptations to their environment [4]. Some of primary metabolic components may serves as a branching point of biosynthetic pathways which leads to the end products of secondary metabolic products. Growth media and incubating conditions have a very strong influence on secondary metabolite production. Secondary metabolism is regulated by the factors like carbon sources, nitrogen sources, phosphate, $\mathrm{NaCl}$, trace elements and different parameters like temperature, $\mathrm{p}^{\mathrm{H}}$ and incubating time intervals [5]. These regulatory factors involves in balancing of biosynthesis and yield of antibiotic activity. Conventional practice of single factor optimization maintains other factors to get involved in improvement of unspecified level to optimum constant level. The study of optimization for secondary metabolite production from marine fungi is to determine the minimum inhibitory concentration of the fungal metabolite against the test pathogens. So our present study is focussed on optimization of antibiotic production by marine fungi.

\section{MATERIALS AND METHODS}

\section{Isolation of marine fungi}

The marine soil sediments are collected from the Nizampatnam mangroves, Guntur district (Andhra Pradesh, India.) are serially diluted with sterile distilled water. Aliquots of $0.2 \mathrm{ml}$ from each sample are spread onto potato dextrose agar plates (containing 20\% potato, $2 \%$ dextrose, $2 \%$ agar). The antibiotics about $50 \mathrm{mg}$ were added to molten media to inhibit the growth of bacteria. Two replicate agar plates are used for each sample. After $5 \mathrm{~d}$ of incubation at $30{ }^{\circ} \mathrm{C}$, the inoculated agar plates were examined daily for the presence of fungal hyphae, using a dissecting microscope at 20x magnification. Distinct fungal colonies on the agar plates were then transferred to new agar plates for further isolation and purification [6].

\section{Basal medium}

According to literature Potato dextrose broth medium is used as a basal medium. The fungal species about $5 \mathrm{~mm}$ mycelial disk obtained from $7 \mathrm{~d}$ old spore culture are inoculated in $100 \mathrm{ml}$ of the medium dispersed in $250 \mathrm{ml}$ sterilized conical flasks and incubated at $27{ }^{\circ} \mathrm{C}$ for $5 \mathrm{~d}$. After incubation the mycelia is harvested by filtration using whatman no 1 filter paper, washed thoroughly with distilled water to remove medium components. Excess of water is removed by blotting with filter papers. The mycelia are allowed to get dry at $50{ }^{\circ} \mathrm{C}$ to express as dry weight (in $\mathrm{mg} / 250 \mathrm{ml}$ ). The production of bioactive metabolites are expressed by measuring the diameter of the inhibition zone against test organisms including Salmonella enterica MTCC 10248, Escherichia coli ATCC 8739, 
Pseudomonas aeruginosa ATCC 27853, Staphylococcus aureus ATCC 25923, Vibrio cholerae ATCC 15748.

\section{Identification of the fungal isolates}

Morphological studies of Mycelia is done by using lacto phenol cotton blue staining to identify genus level [7]. The effects of salt concentration on the growth of isolated fungi were determined by Joshi et al., 2008 [8]. The selected strains are cultured with potato dextrose Broth medium at $27^{\circ} \mathrm{C}$ for $5 \mathrm{~d}$.

\section{Production of antimicrobial substances from marine fungi}

All the potent fungal isolates were inoculated in to each potato dextrose broth and incubated at $27{ }^{\circ} \mathrm{C}$ for $5 \mathrm{~d}$. Then biomass is removed aseptically using sterile spatula, and crude was extracted by using double amount (1:1) of chemical solvent (ethyl acetate) for $24 \mathrm{~h}$. The organic solvent layer was collected and concentrated by evaporation. The concentrated crude extract was collected for antibacterial assay.

\section{Antimicrobial activity}

Antimicrobial activity of marine fungal crude extracts was tested by using agar well diffusion method [9]. A $50 \mu \mathrm{l}$ of crude extract from stock solution is added in to each well ( $6 \mathrm{~mm}$ diameter). The crude extract diffused in to nutrient agar plates inoculated with test organisms (Salmonella enterica MTCC 10248, Escherichia coli ATCC 8739, Pseudomonas aeruginosa ATCC 27853, Staphylococcus aureus ATCC 25923, Vibrio cholera ATCC 15748). All the plates are incubated at $37^{\circ} \mathrm{C}$ for $24 \mathrm{~h}$. The zone of inhibition was measured and expressed diameter was in millimetre.

\section{Minimum inhibitory concentrations (MIC)}

MIC of secondary metabolite was performed by agar-cup diffusion method. The crude extract was dissolved in different concentrations ranging from highest concentration of $10,000 \mu \mathrm{g} / \mathrm{ml}$ and then dilution were performed at concentration of $5000 \mu \mathrm{g} / \mathrm{ml}, 4000$ $\mu \mathrm{g} / \mathrm{ml}, 3500 \mu \mathrm{g} / \mathrm{ml}, 3000 \mu \mathrm{g} / \mathrm{ml}, 2500 \mu \mathrm{g} / \mathrm{ml}, 2000 \mu \mathrm{g} / \mathrm{ml}, 1500$ $\mu \mathrm{g} / \mathrm{ml}, 1000 \mu \mathrm{g} / \mathrm{ml}, 750 \mu \mathrm{g} / \mathrm{ml}, 500 \mu \mathrm{g} / \mathrm{ml}$ and $250 \mu \mathrm{g} / \mathrm{ml}$. MIC value of marine fungal crude extract was determined against five different human pathogens belonging to both gram positive and gram negative groups such as Salmonella enterica MTCC 10248, Escherichia coli ATCC 8739, Pseudomonas aeruginosa ATCC 27853, Staphylococcus aureus ATCC 25923, Vibrio cholera ATCC 15748. Bacterial suspensions of the test organism were prepared in sterilized Mueller-Hinton broth. Then $50 \mu$ of the extract was added to each well. Culture medium without samples and others without microorganisms were used in the tests as positive and negative controls. Plates were incubated at $37{ }^{\circ} \mathrm{C}$ for $20-24 \mathrm{~h}$ and MIC was measured by zone of inhibition [18].

\section{Optimization of growth and active metabolite production}

Optimization for the production of bioactive secondary metabolites was made with potato dextrose broth and these studies are used to estimate the potency of the compound by antimicrobial assay to check the activity of the compound from marine fungi. These studies are useful distinguish the effect of physical and chemical parameters on the growth and bioactive metabolite. So, these optimization studies are carried out through each parameter and its dependence on the other parameter on the biomass and metabolite. The optimization studies were done as following.

\section{Effect of optimization on mycelia and bioactive metabolite production}

Effect of culture media on mycelia growth and bioactive metabolite production

To select a suitable growth medium for marine fungal isolates (gaps1 and gaps-2) are grown in different culture media such as Potato dextrose broth, Czapek's Dox broth, Sabouraud dextrose broth, Malt extract broth and Peptone Yeast Glucose broth. The medium which exhibits maximum biomass accumulation and bioactive metabolite (antibiotic) production in terms of zone of inhibition was used as the optimized medium for further study. All the selected media compositions are Hi-media procured from Hi-Media Laboratories, Mumbai, India.

\section{Effect of agitation on mycelia growth and bioactive metabolite production}

Effect of agitation on mycelia growth and bioactive metabolite production was studied by incubating culture in conical flasks on an obituary shaker incubator at different $\operatorname{rpm}(50,100,150$ and 200 $\mathrm{rpm}$ ). Control experiment was done by incubating the inoculated media at static condition. The metabolite production of the inoculated media was determined after seven days of incubation at $280 \mathrm{C}$ at $150 \mathrm{rpm}$ in an orbital shaker. After incubation the mycelia growth as well as the bioactive metabolite production for each rpm concentration was estimated and recorded.

\section{Effect of salinity on mycelia growth and bioactive metabolite} production

The effect of salinity on mycelia growth and bioactive metabolite produced by the isolates were carried out by incubating in various $\mathrm{NaCl}$ concentrations (3\%-7\%) at room temperature with optimum incubation time period. The biomass as well as the bioactive metabolite production for each sodium chloride concentration were estimated and recorded.

Effect of incubation time on mycelia growth and bioactive metabolite production

To study the effect of incubation time (h 24-240) $100 \mathrm{ml}$ of potato dextrose broth flasks were inoculated with $5 \mathrm{~mm}$ mycelia disks of seven days old fungal culture under aseptic condition and incubated. The mycelia weight and antimicrobial production were recorded at the end of the incubation period [10].

\section{Effect of $\mathrm{pH}$ on mycelia growth and bioactive metabolite production}

The effect of $\mathrm{pH}$ on the mycelia growth and bioactive metabolite production of the isolates were tested by using liquid cultures containing different $\mathrm{pH}$ levels (pH 4-9). $100 \mathrm{ml}$ of liquid medium was poured into a $250 \mathrm{ml}$ conical flask under aseptic conditions along with optimized parameters. The medium was adjusted to the desired $\mathrm{pH}$ by adding $1 \mathrm{~N} \mathrm{NaOH}$ [11]. Flasks were sterilized at $121^{\circ} \mathrm{C}$ at 15 psi for 20 min. Each flask was inoculated with $5 \mathrm{~mm}$ diameter mycelia disc in sterile conditions. Inoculated flasks were incubated at $27 \pm 1{ }^{\circ} \mathrm{C}$ for seven days and the dry mycelia weight and bioactive metabolite productions were recorded.

\section{Effect of temperature on mycelia growth and bioactive metabolite production}

The fungal isolates were subjected to different temperature (from 15 ${ }^{\circ} \mathrm{C}$ to $45^{\circ} \mathrm{C}$ ) with parameters like salinity, incubation and $\mathrm{pH}$ to study the optimum temperature required for mycelia growth and bioactive metabolite yield. $100 \mathrm{ml}$ of basal medium is prepared and sterilized at $121{ }^{\circ} \mathrm{C}$ at $15 \mathrm{psi}$ for $20 \mathrm{~min}$. Under aseptic conditions $5 \mathrm{~mm}$ diameter of the culture discs were inoculated and incubated for seven days. After incubation the dry mycelial weight and the antimicrobial productions were recorded [12].

\section{Effect of carbon sources on mycelia growth and bioactive metabolite production}

To study the effect of different carbon sources (glucose, lactose, sucrose, fructose, galactose, xylose, maltose, arabinose, raffinose) about $1 \%$ of each is added to the selected medium individually. Each flask with different carbon sources were inoculated with a $5 \mathrm{~mm}$ mycelia disc of three days old fungal cultures and incubated for seven days. After incubation period biomass (mycelia weight) and the production of bioactive metabolites are recorded [13]

Effect of nitrogen source on mycelia growth and bioactive metabolite production

To study the effect of different nitrogen sources (beef extract, yeast extract, peptone, ammonium chloride and sodium nitrate) are used about $1 \%$ of each to the selected medium individually and the dextrose was used as the source of carbon in all the treatments. Flasks were inoculated with $5 \mathrm{~mm}$ mycelia disks of seven days old 
fungal culture under aseptic condition and incubated for seven days. The mycelia weight and antimicrobial production were recorded at the end of the incubation period [14-17].

Effect of micro nutrients on mycelia growth and bioactive metabolite production

To study the effect of mineral salts (trace elements) like $\mathrm{CaCl}_{2}, \mathrm{FeSO}_{4}$, $\mathrm{MnCl}_{2}, \mathrm{MgSO}_{4}, \mathrm{ZnCl}_{2}$,

$\mathrm{K}_{2} \mathrm{HPO}_{4}$, are used about $0.01 \%$ of each to the selected medium individually and the dextrose was used as the source of carbon and nitrogen sources in all the treatments. Flasks were inoculated with 5 $\mathrm{mm}$ mycelia disks of seven days old fungal culture and incubated. The mycelia weight and antimicrobial production were recorded.

\section{RESULTS AND DISCUSSION}

Isolation and morphological characterization of marine fungi

Totally 43 fungal isolates were recovered, among these 10 morphologically different strains were sub cultured and maintained for further analysis. According to Microscopic and Cultural characteristics the isolates were belonging to Aspergillus species (date not disclosed) were found predominantly in the marine soil from Nizampatnam, Guntur district (Andhra Pradesh).

\section{Extraction of secondary metabolite}

Extraction of the secondary metabolite was effectively done with ethyle acetate and broth culture in ratio 1:1, and allowed to vacuum drying. The extracted compound was stored in the same $1 \mathrm{ml}$ of solvent and stored for further analysis.

\section{Media optimization}

Production of growth and secondary metabolite from marine fungi strongly influenced by parameters like salinity, temperature, $\mathrm{pH}$, incubation time and media components, carbon, nitrogen sources and trace elements. It is significant in order to study the influence of various physico-chemical parameters to find out optimized media for the production of potent secondary metabolite.

In present study, initially one parameter was assessed and it was then incorporated at its optimized level in the subsequent experiments using potato dextrose broth medium.
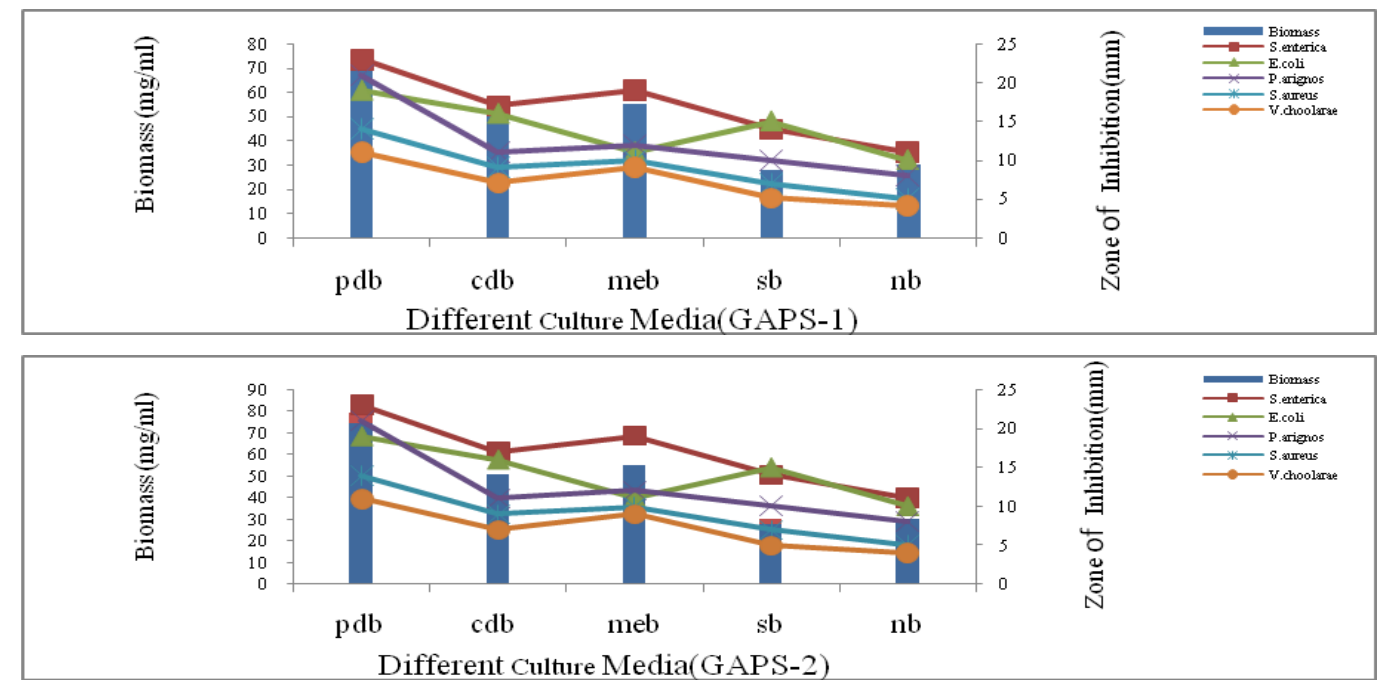

Fig. 1: Effect of culture media on microbial growth on marine fungi

Effect of Culture media on Secondary metabolite production and biomass by gaps- 1 , gaps- 2 showing maximum growth and antibiotic production in Potato dextrose broth comparatively to other media. Fig. 1 Reveals that the effect of different growth media on biomass and bioactive metabolite production of inoculated gaps- 1 and gaps- 2 within $6 \mathrm{~d}$ of incubation (on orbital shaker incubator). According to fig. 1 among the tested media, maximum mycelial dry weight and bioactive metabolite production was recorded in potato dextrose broth medium.
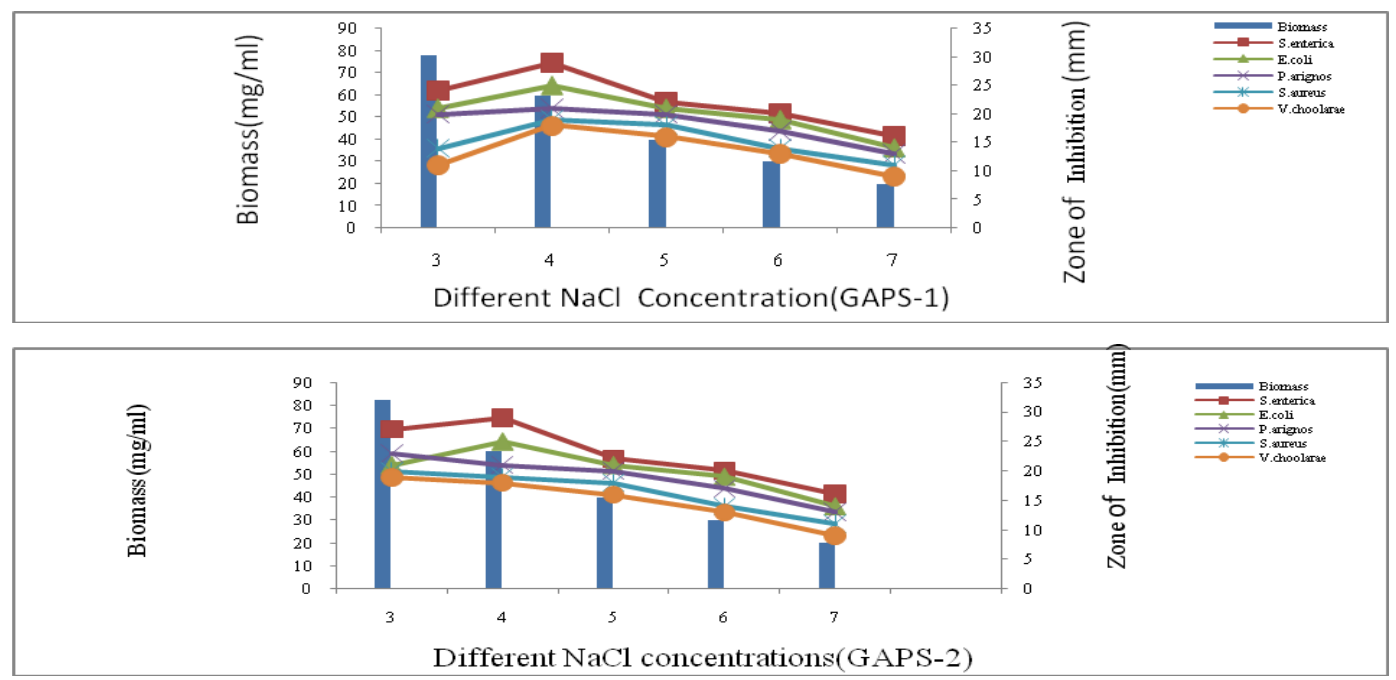

Fig. 2: Effect of NaCl concentration on microbial growth on marine fungi 
Effect of Culture media on Secondary metabolite production and biomass by gaps-1, gaps-2 showing maximum growth and antibiotic production in Potato dextrose broth comparatively to other media. Fig.
2 Reveals that the effect of Nacl on Secondary metabolite production and biomass by gaps- 1 and gaps-2showing maximum growth and antibiotic production in pdb at $\mathrm{Nacl} 3 \%$ with agitation $150 \mathrm{rpm}$.
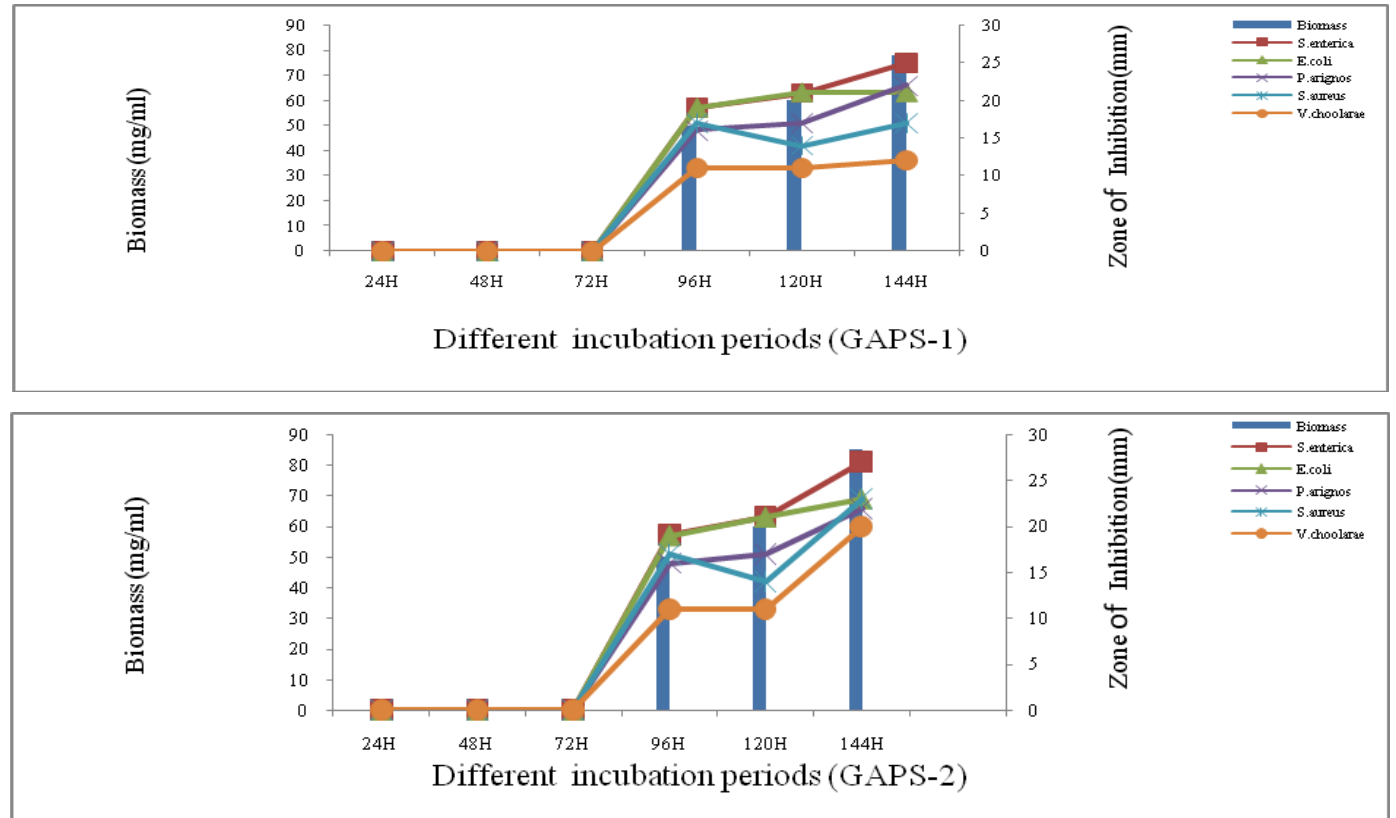

Fig. 3: Effect of incubation on microbial growth of marine fungi

Fig. 3 reveals that the effect of incubation period on biomass and bioactive metabolite production of inoculated gaps 1 and gaps 2 within $6 \mathrm{~d}$
Effect of incubation time period on Secondary metabolite production and biomass by GAPS 1 and 2 showing maximum growth and antibiotic production in pdb at Nacl 3\% with incubation time $144 \mathrm{~h}$
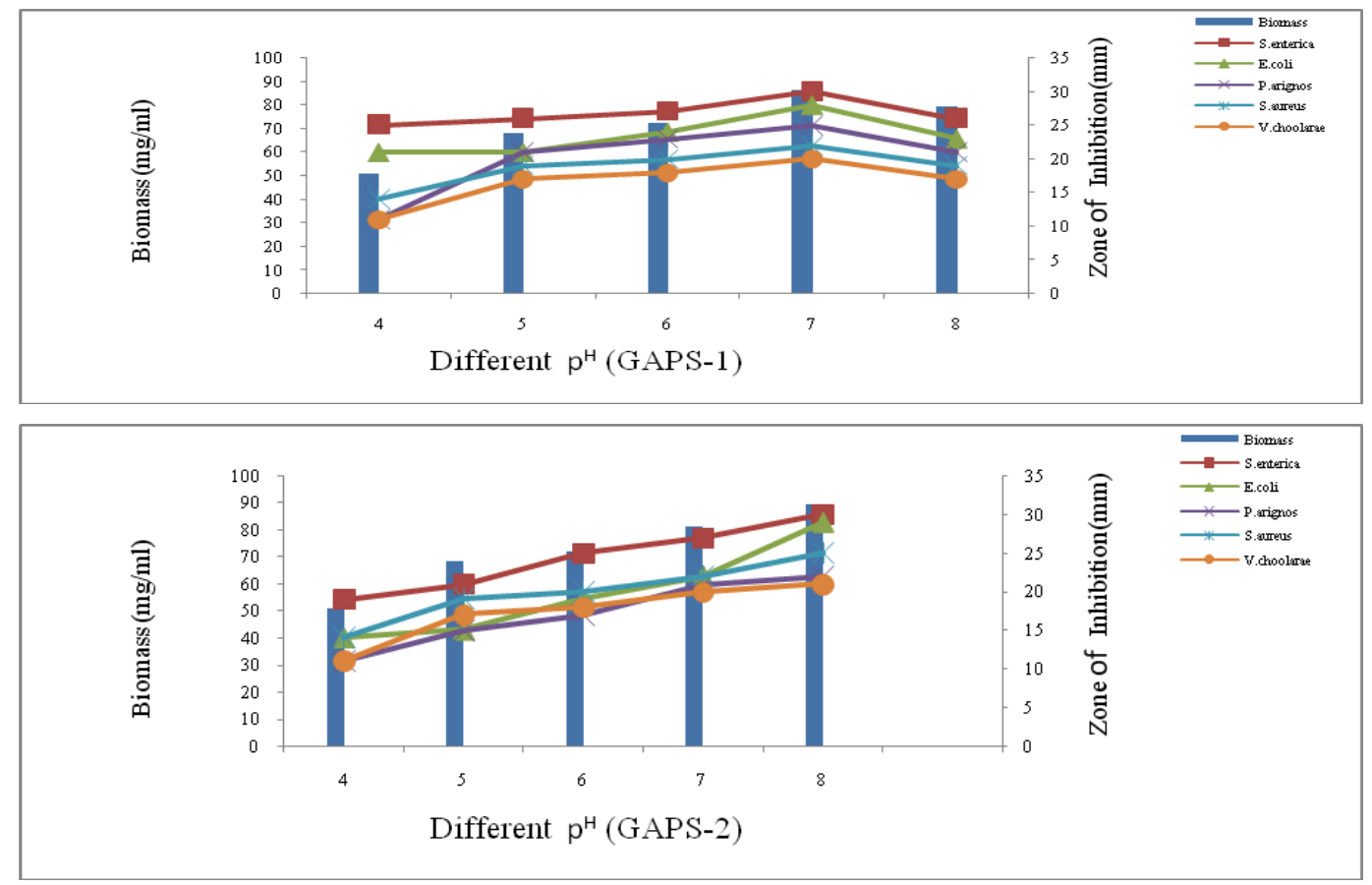

Fig. 4: Effect of $\mathrm{p}^{\mathrm{H}}$ on microbial growth of marine fungi

Fig. 4 explains about effect of $\mathrm{P}^{\mathrm{H}}$ on Secondary metabolite production and biomass by gaps 1 and 2 showing maximum growth and antibiotic production in pdb at Nacl 3\%; incubation time $144 \mathrm{~h}$; $\mathrm{PH}_{-}$-7(GAPS-1), $8(G A P S-2)$. Selected potential marin fungal metabolite were subjected to various concentrations of $\mathrm{pH}$ ranging from 3.0 to 10.0 were taken for the study of an interval of $\mathrm{pH}$ 1.0. Maximum growth at ph 7.0 (gaps 1) ph 8.0 (gaps-2) and decreased further increases the $\mathrm{pH}$ beyond 7.0. No growth was registered at ph range below 4 . 

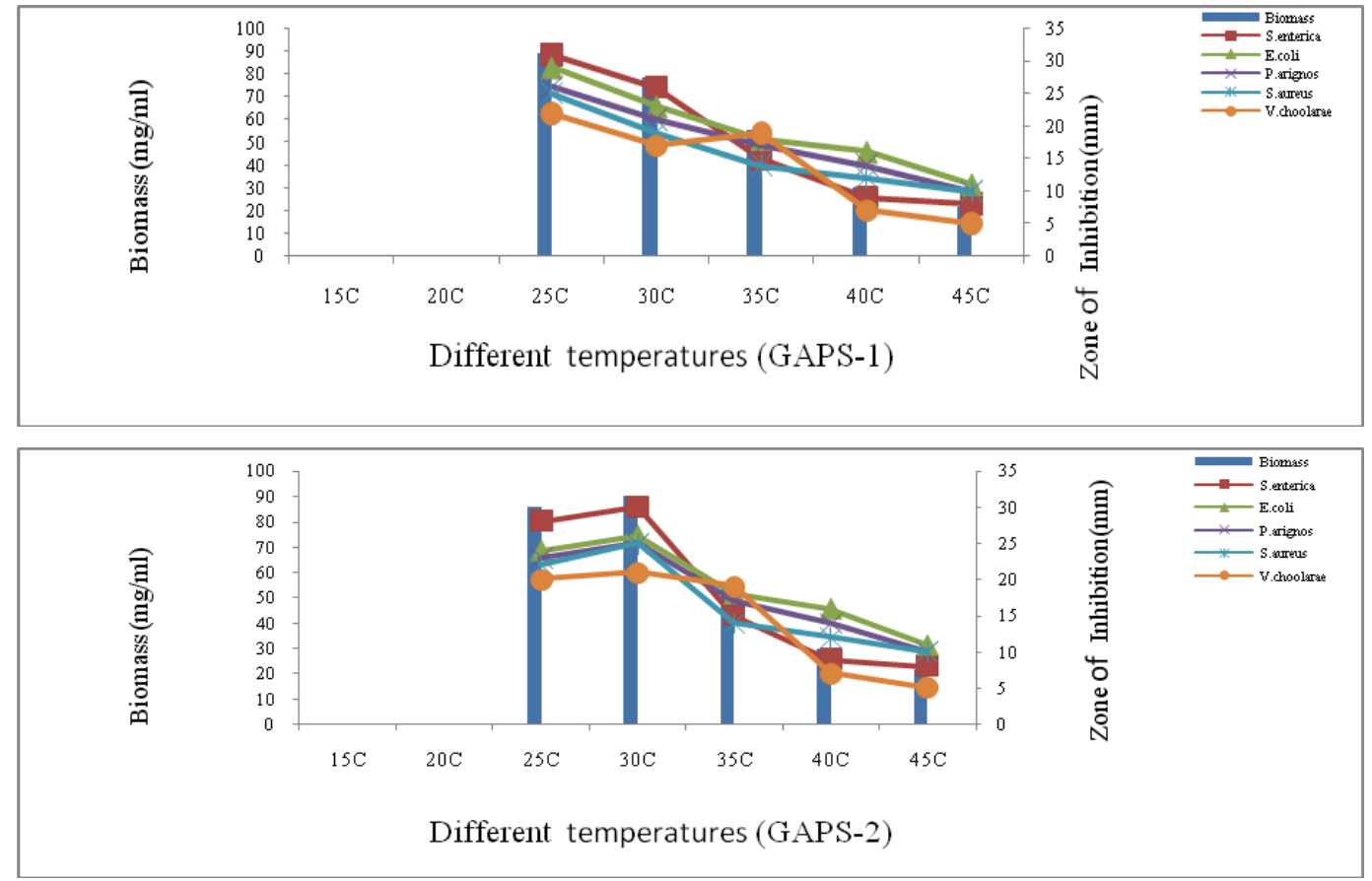

Fig. 5: Effect of temperature on microbial growth on marine fungi

From fig. 5 effect of Temperature on Secondary metabolite production and biomass by gaps-1, gaps-2 showing maximum growth and antibiotic production in PDB at Nacl 3\%; incubation time
144 h; PH-7(GAPS-1), 8(GAPS-2); temperature-25 ${ }^{\circ} \mathrm{C}(\mathrm{GAPS}-1), 30$ ${ }^{\circ} \mathrm{C}(\mathrm{GAPS}-2)$ The requirements for specific physical temperature $\left(250 \mathrm{c}\right.$ and $\left.30^{\circ} \mathrm{C}\right)$.
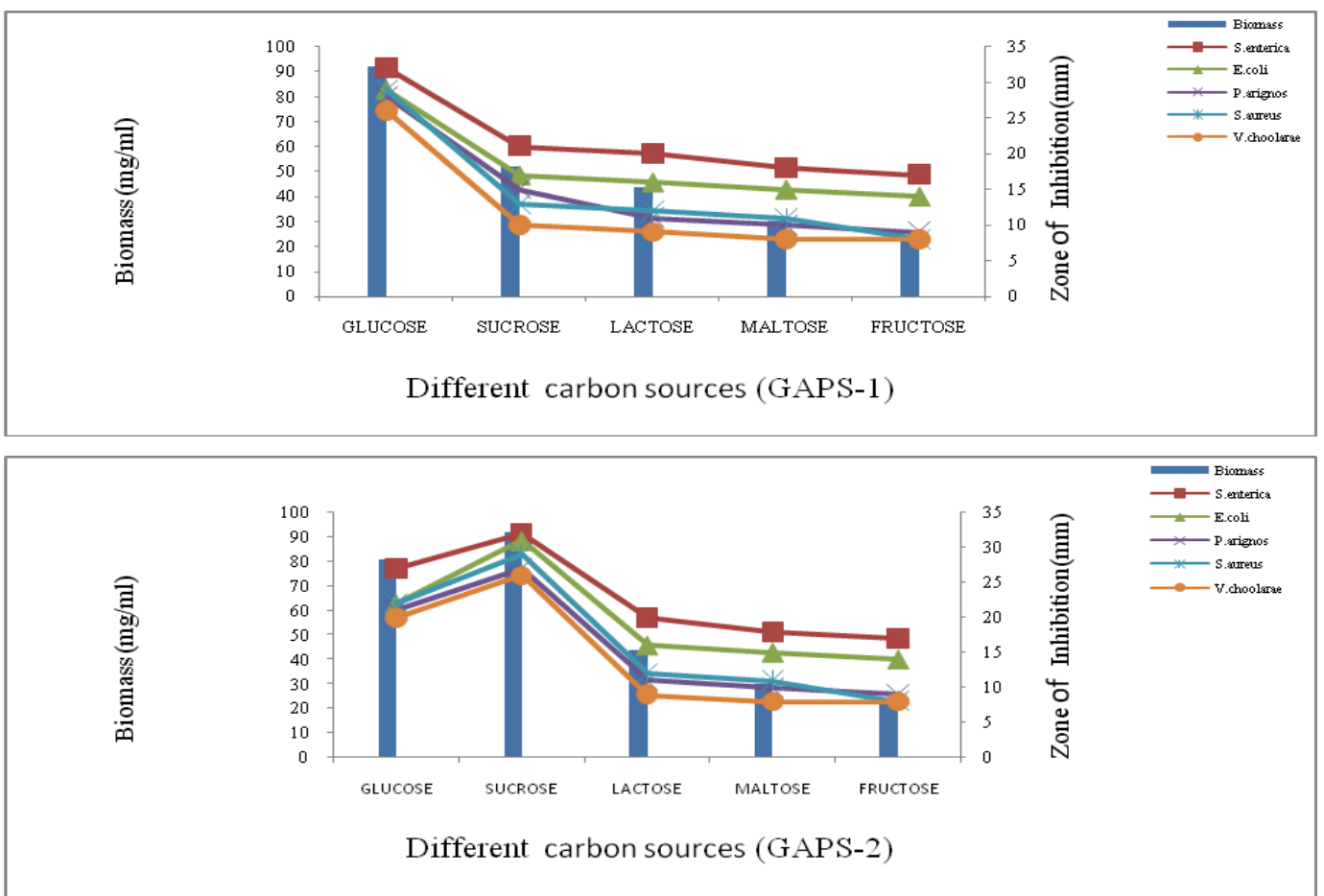

Fig. 6: Effect of carbon sources on microbial growth on marine fungi

Effect of carbon sources on Secondary metabolite production and biomass by gaps-1,gaps- 2 showing maximum growth and antibiotic production in pdb at Nacl 3\%; incubation time $144 \mathrm{~h}$; $\mathrm{PH}_{-}$-7(GAPS-1) 8(GAPS-2); temperature- $25{ }^{\circ} \mathrm{C}$ (GAPS-1), $30{ }^{\circ} \mathrm{C}$ (GAPS-2). $1 \%$ of carbon sources: Glucose (GAPS-1), sucrose (GAPS-2). From fig. 6 of all the tested carbon sources $(1 \% \mathrm{w} / \mathrm{v})$ in the selected medium inoculated with gaps-1 and gaps- 2 incubated shaken for $6 \mathrm{~d}$, glucose and sucrose supported the highest level of mycelia growth and bioactive metabolite production. 

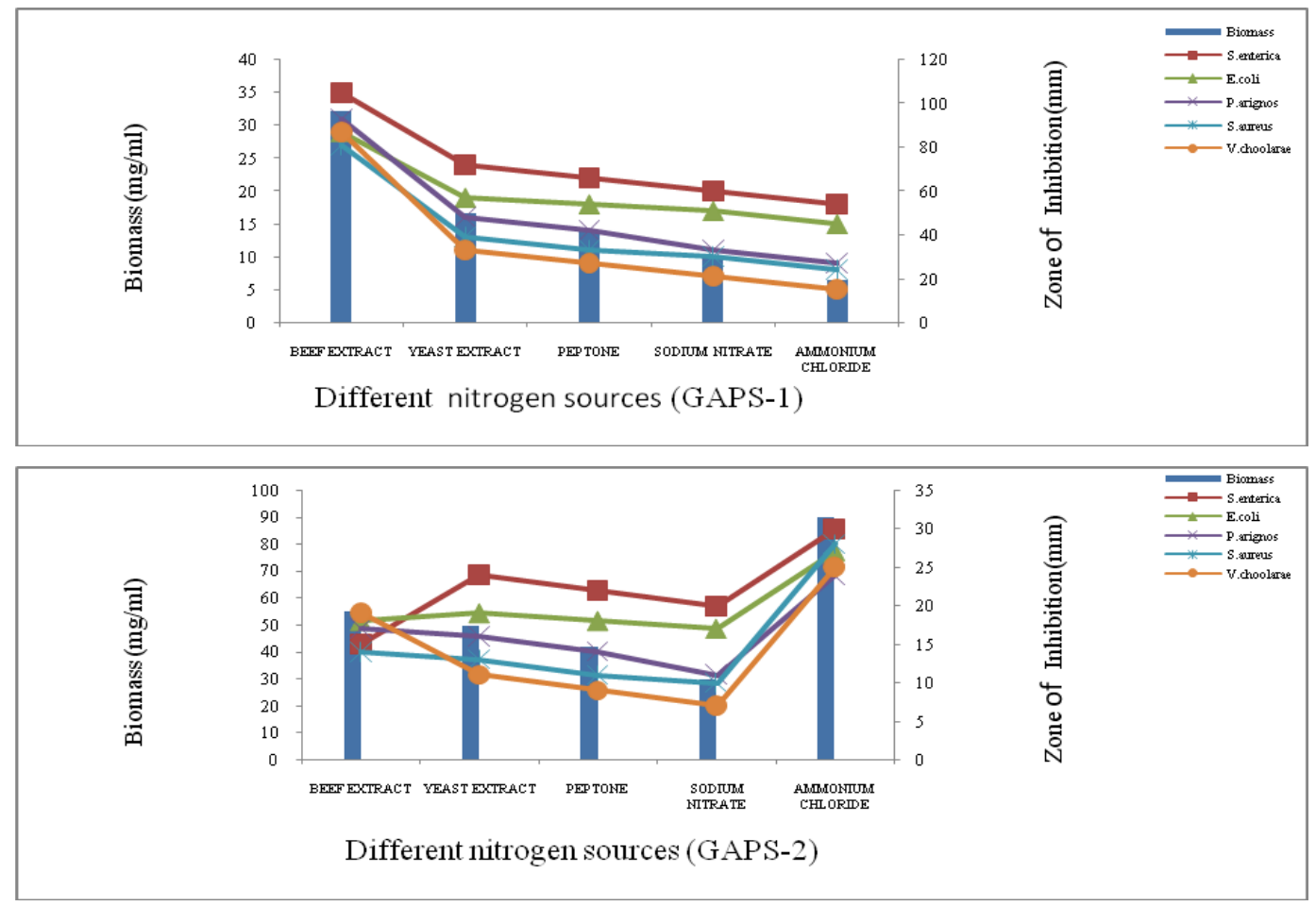

Fig. 7: Effect of nitrogen sources on microbial Growth on marine fungi

According to fig. 7 effect of nitrogen sources on Secondary metabolite production and biomass by GAPS-1and2showing maximum growth and antibiotic production in pdb at Nacl 3\%; incubation time $144 \mathrm{~h}$; $\mathrm{PH}_{-}$ 7(GAPS-1), 8(GAPS-2); temperature- $25^{\circ} \mathrm{C}$ (GAPS-1), $30{ }^{\circ} \mathrm{C}$ (GAPS-2), $1 \%$ of carbon sources: Glucose(GAPS-1), sucrose (GAPS-2); $1 \%$ of nitrogen sources: Beef extract(GAPS-1), Ammonium chloride(GAPS-2) Addition of equimolar amounts of various nitrogen sources in the selected medium supplemented with $1.0 \%(\mathrm{w} / \mathrm{v})$ show that beef extract and ammonium sulphate were good nitrogen sources on the mycelia growth and bioactive metabolite production
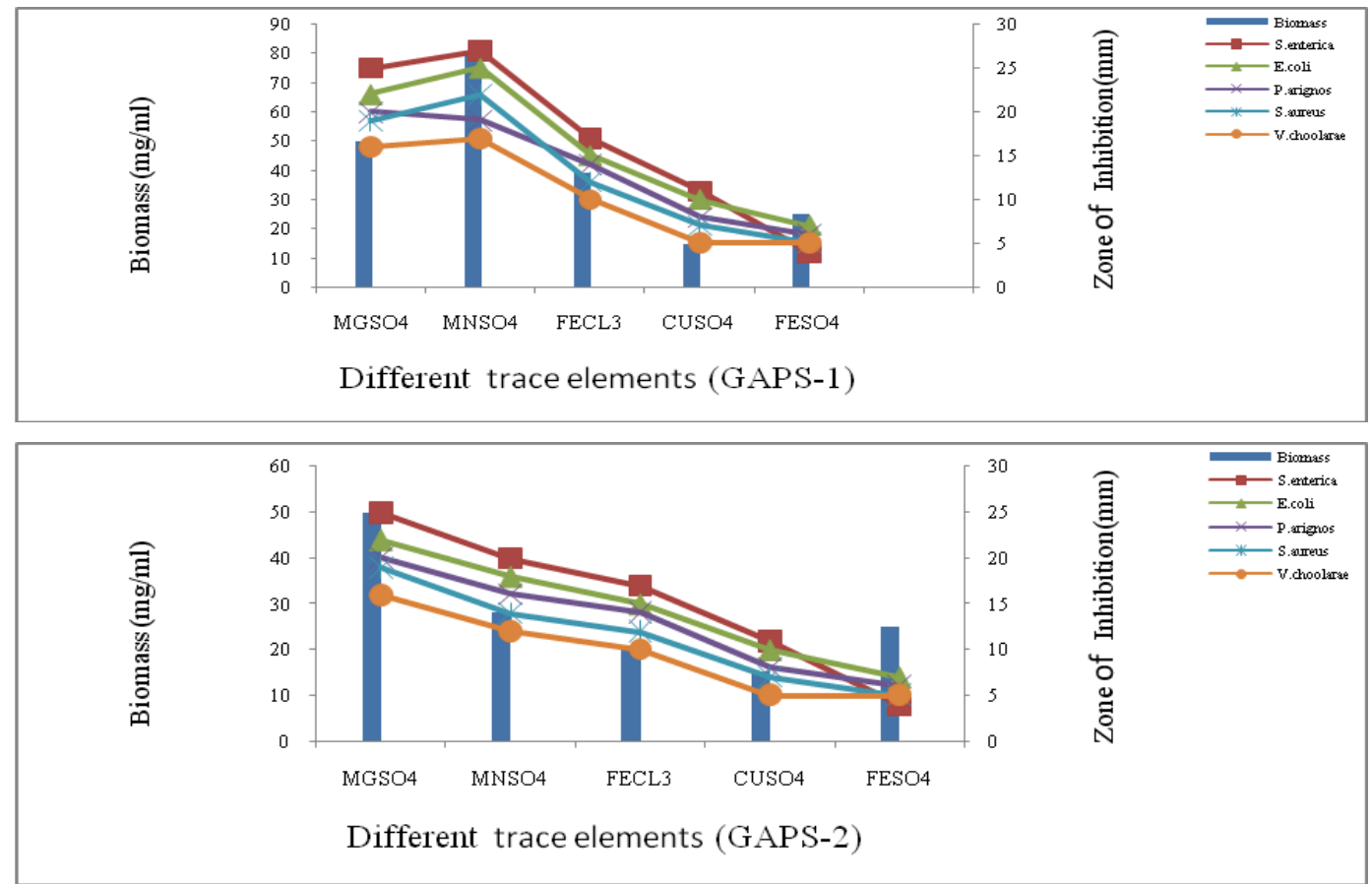

Fig. 8: Effect of trace elements on microbial growth on marine fungi

Fig. 8 shows the effect of trace elements on Secondary metabolite production and biomass by gaps-1, gaps- 2 showing maximum growth and antibiotic production in pdb at Nacl 3\%; incubation time
$144 \mathrm{~h}$; $\mathrm{PH}^{\mathrm{H}}$-7(GAPS-1), 8(GAPS-2); temperature-25 ${ }^{\circ} \mathrm{C}$ (GAPS-1), 30 ${ }^{\circ} \mathrm{C}$ (GAPS-2) $1 \%$ of carbon sources: Glucose(GAPS-1), sucrose (GAPS2); $1 \%$ of nitrogen sources: Beef extract (GAPS-1), Ammonium 
chloride(GAPS-2) but trace elements shown the effect on growth and antibiotic production by reducing the activity of gaps-1, gaps- 2 .

\section{Antibacterial activity and minimum inhibitory concentration}

Antimicrobial resistance had become a serious threat in treating of infectious diseases, to overcome this problem many academicians and scientists are search of antimicrobials against the clinical pathogens from marine environments. The present study also can be useful for the survey of antimicrobials from marine fungi. In this present study about 43 isolates were isolated from the marine soil samples which act as antibacterial against the pathogens like Salmonella enterica MTCC 10248, Escherichia coli ATCC 8739, Pseudomonas aeruginosa ATCC 27853, Staphylococcus aureus ATCC 25923, Vibrio cholera ATCC 15748. Among these isolates 10 strains were selected as they have morphological variations. About 5 samples were collected with highest antibacterial activity and the minimum concentration ranges from 250 $\mu \mathrm{g} / \mathrm{ml}$ to $10000 \mu \mathrm{g} / \mathrm{ml}$ but the sample gaps-1 had more activity 250 $\mu \mathrm{g} / \mathrm{ml}$ than gaps $-2300 \mu \mathrm{g} / \mathrm{ml}$ table 1 .

Table 1: Minimum inhibitory concentration

\begin{tabular}{lll}
\hline Pathogens & Mic value $(\boldsymbol{\mu g} / \mathbf{m l})$ Gaps-1 & Mic value $(\boldsymbol{\mu g} / \mathbf{m l})$ Gaps-2 \\
\hline Salmonella enterica & 1500 & 1500 \\
E. coli & 250 & 300 \\
Pseudomonas aeruginosa & 1000 & 1000 \\
Staphylococcus aureus & 1500 & 1500 \\
Vibrio cholerae & 1500 & 1500 \\
\hline
\end{tabular}

According to the above figures, strains are having high microbial activity when compared with streptomycin as standard. The factor dependent optimization shows that there is a great effect of different types of parameters on the marine fungal growth and production of metabolites against bacterial pathogens. The parameters like $\mathrm{p}^{\mathrm{H}}$, temperature, incubation period, macro and micronutrients. In the present study role of trace elements like magnesium, manganese, ferrous and zinc had less metabolite production when correlate with other parameters. The stability of metabolite seems to be about five days and maximum activity is within $24 \mathrm{~h}$ of time.

\section{CONCLUSION}

The optimization Process of present study concludes that optimum conditions are required for the production of bioactive metabolite from marine fungi gaps- 1 and gaps- 2 . The optimum conditions of the marine fungi were incubation period $144 \mathrm{~h} \mathrm{pH} 8.0$ and 7.0 and temperature $25^{\circ} \mathrm{C}$ and $30^{\circ} \mathrm{C}$. Aeration also played a crucial role in terms of product formation. Agitation speed of $150 \mathrm{rpm}$ showed maximum yield. Secondary metabolites had showed broad spectrum activity against bacteria. Hence these bioactive metabolites can be used as source of antimicrobial. Further studies of these metabolites will be carried on purification, characterization and identification of bioactive metabolites of marine fungi.

\section{ACKNOWLEDGEMENT}

The author will be always thankful to Dr. P. Sudhakar for his guidance to carry out the present work and also my sincere acknowledge to the UGC-RGNF for the financial support.

\section{CONFLICT OF INTERESTS}

Declare none

\section{REFERENCES}

1. Zhang J, Li YT, Yong JL, Li MC, Yan JM, Li SZ, et al. Anthracenedione derivatives as anticancer agents isolated from secondary metabolites of the mangrove endophytic fungi. Mar Drugs 2010;8:1469-81.

2. S Omura. Philosophy of new drug discovery. Microbiol Rev 1986;50:259-79.

3. JF Martin, AL Demain. Control of antibiotic synthesis. Microbiol Rev 1980;44:230-51.

4. Padmavathi T, Nandy V, Agarwal P. Optimization of the medium for the production of cellulases by Aspergillus terreus and Mucor plumbeus Eur J Exp Biol 2012;2:1161-70.

5. Betina V. Bioactive secondary metabolites of microorganisms progress in industrial microbiology, Elsevier. Amsterdam; 1994. p. 30.

6. Hairong Xiong, Shuhua Qi, Ying Xu, Li Miao, Pei-Yuan Qian. Antibiotic and antifouling compound production by the marinederived fungus cladosporium sp. F14. J Hydro Environ Res 2009;2:264-70.
7. Yin Lu, Chuan Chen, Hong Chen, Jianfen Zhang. Isolation and identification of endophytic fungi from actinidia macrosperma and investigation of their bioactivities. J Evidence Based Complementary Altern Med 2012;8. http://dx.doi.org/ 10.1155/2012/382742

8. Joshi F, Chaudhari A, Joglekar P, Archana G, Desai AJ. Effect of expression of $\mathrm{B}$ radyrhizobium japonicum $61 \mathrm{~A} 152 \mathrm{feg}$ a gene in mesorhizobium sp., on its competitive survival and nodule occupancy on Arachis hypogeal. Appl Soil Ecol 2008;40:338-47.

9. NCCLS-National Committee for Clinical Laboratory Standards: Performance standards for antimicrobial disc susceptibility tests. 8th edition. Approved Standard, document M2-A8[S]. NCCLS, Wayne, Pennsylvania; 2003.

10. Majumdar Mk, Majumdar Sk. Effects of minerals on neomycin production by streptomyces fradiae. Appl Microbiol 1965;13:190-3.

11. Singh LS, Mazumder S, Bora TC. Optimisation of process parameters for growth and bioactive metabolite produced by a salt-tolerant and alkaliphilic actinomycete, Streptomyces tanashiensis strain A2D. J Mycol Med 2009;19:225-33.

12. Ripa FA, Nikkon F, Zaman S, Khondkar P. Optimal conditions for antimicrobial metabolites production from a new streptomyces sp. RUPA-08PR isolated from Bangladeshi soil. Mycobiology 2009;37:211-4.

13. Naik MK, Hiremath PC, Hegde RK. Physiological and nutritional studies on Colletotrichum gloeosporioides, a causal agent of anthracnose of beetlevine, Mysore. J Agric Sci 1988;22:471-4.

14. Thakur D, Bora TC, Bordoloi GN, Maiumdar S. Influence of nutrition and culturing conditions for optimum growth and antimicrobial metabolite production by Streptomyces sp. 201. J Mycol Med 2009;19:161-7.

15. Kumara KLW, Rawal RD. Influence of carbon، nitrogen، temperature and $\mathrm{pH}$ on the growth and sporulation of some Indian isolates of C. gloeosporioides causing anthracnose disease of papaya (Carrica papaya L.). Tropical Agricul Res Extension 2008;11:7-12.

16. Miao L, Kwong TFN, Qian PY. Effect of culture conditions on mycelial growth, antibacterial activity, and metabolite profiles of the marine-derived fungus arthrinium c. f. saccharicola. Appl Microbiol Biotechnol 2006;72:1063-73.

17. Gautam SP, Bundela PS, Pandey AK, Awasthi MK, Sarsaiya S. Optimization of the medium for the production of cellulase by the Trichoderma viride using submerged fermentation. Int J Environ Sci 2010;1:4.

18. Saha A, Rahman MS. Antimicrobial activity of crude extract from Calycopterisfloribunsa. Bang J Microbiol 2008;25:137-9.

\section{How to cite this article}

- Anuhya G, Jyostna V, Aswanikumar YVV, Bodaiah B, Sudhakar P. Influence of physico-chemical parameters on secondary metabolite production by marine fungi. Int J Curr Pharm Res 2017;9(5):112-118. 